\title{
A reliable and accurate system of joint position sense measurement.
}

\author{
Rabiya Noor, Gholamreza Olyaei*, Mohammad Reza Hadian, Saeed Talebian, Muhammad Salman \\ Bashir
}

School of Rehabilitation, Brain and Spinal Injury Research Center, Tehran University of Medical Sciences-International Campus (IC-TUMS), Iran

\begin{abstract}
Objective: Measurement of range of motion is used to measure the joint Position Sense (JPS). The objective of this study was to give an easy and less costly method of measuring JPS.

Methods: The study conducted at Tehran University of Medical Sciences, in Iran in May 2017. In this study researcher used the system of digital photographs, non-reflective markers and software for measuring the accuracy and reliability. The fixed arm of the goniometer was horizontal. The moveable arm was positioned at the $15^{\circ}$ initially and then selected it at different degrees $(30,45,60$ and 75$)$ randomly. 5 reference angles were selected from $15^{\circ}$ to $75^{\circ}$. By using moving arm three photos were taken from each angle. Reliability and validity of measurement for each angle was disclosed by utilizing T-test, correlation (reliability test) and $\mathbf{R}$ square of regression (validity test). Also relative and absolute error is used to evaluate the accuracy of this method.

Results: Results showed that mean $\left(R^{\wedge} 2\right)$ for validity is 0.99 , T-test p-value for reliability is 0.41 it means there is no significant difference between these methods and for correlation (reliability) mean value was 0.97 and averages of absolute error and relative error were (-0.44 and 0.62$)$ respectively.

Conclusion: It was disclosed that method comprising of digital photography non-reflective markers showed similar results as compared to goniometer in terms of reliability, validity and accuracy of this method. In conclusion, it was a novel approach to measure JPS with salient features of user friendly, fast, less expensive, accurate and reliable.
\end{abstract}

Keywords: Joint position sense, Range of motion, Photography.

Accepted on April 05, 2018

\section{Introduction}

Angular measurement has been used to measure Joint Position Sense [1]. Different method used to check the angle measurement such as electric goniometer and digital photographs [2,3]. Electrogoniometer cannot be used for all body because it may receive abnormal sensory feedback [1]. It can also be affected by the pressure and straps which create less sensory information [4]. In this study universal goniometer is used which is more reliable than electronic goniometer [5]. In computer using method for analysis of JPS, angles were measured through digital cameras, video cameras and digitizing system which is expensive and difficult to operate [3]. In some system infrared was used to identify the angle [6]. In kinematic researches the main focus was on the motion analysis which is not the cause. In other words the measurements were taken by taking videos of moving object or body segments [7].

Very less researches were present regarding the method of measuring JPS by using goniometer, photographs [8]. Skin markers have no issue with restriction of movement and usage is simple [9]. Goniometric measurement of the angle with video films is sufficiently precise and some studies used goniometer and photographs to measure JPS [10-12]. But taking photos and then taking prints of those photos to measure JPS was costly method as Herrington used the method to measure knee JPS [10]. Although, angle measurement is easy by using these two methods, their accuracy and reliability is not clear. The objective of this study was to explore the issue: 1) Evaluating the method comprising of non-reflective markers, Microsoft excel software and digital photography in terms of accuracy and reliability of angles measurement.

\section{Materials and Methods}

An innovative study to design a reliable and accurate system of joint position sense measurement was conducted at Tehran University of Medical Sciences, international campus in May 2017.

This research was following the method of Linden et al. which was parallel to Scholz et al. researcher used the system of digital photographs, non-reflective markers and software for measuring the accuracy and reliability [13-15]. Square markers were used for capturing the goniometer ends and scale for 
calibration. Goniometer attached on board and ends were prominent by red markers. The fixed arm of the goniometer was horizontal. The moveable arm was positioned at the $15^{\circ}$ initially and then set it at different degrees (30, 45, 60 and 75) randomly.

Digital video camera (Sony 16.1 M pixels) was positioned at $90 \mathrm{~cm}$ far from the board and raised $80 \mathrm{~cm}$ from the ground, with its lens directing in the direction of the center of the board. Five reference angles were selected from $15^{\circ}$ to $75^{\circ}$. By using the moving arm three photos were taken from each angle. Then the alteration in each angle was measured with the help of self-made excel software.

After that the pictures were transported to computer and imported in the computer based application "Paint" in which researcher noted the position of $\mathrm{X}$ and $\mathrm{Y}$ axis and shifted these values in self-made Microsoft Excel software which gave the value of T-test, correlation between three trials (3 repetition at 15-75 degrees) and $\mathrm{R}$ square regression between average of three repetition and selected goniometer at different degrees $\left(15^{\circ}-75^{\circ}\right)$.

Absolute Error (AE) is operationally defined as the absolute difference between the target angle and the perceived angle. $\mathrm{AE}$ is calculated for each of the 3 trials for the 3 target angles for each limb of each subject [16].
The Relative Error (RE) represents the variability of the errors between trials and indicates the consistency of proprioceptive performance (Figure 1) [16].

\section{Data analysis}

Reliability and validity of measurement of each angle was disclosed by utilizing T-test, correlation (reliability test) and $\mathrm{R}$ square of regression (validity test). Also relative and absolute errors were used to evaluate the accuracy of this method.

Correlation (intrarater reliability) of these two methods manual and Excel Software analysis was found that by using Pearson product moment correlation coefficient. According to above analysis value it was disclosed that method comprising of nonreflective markers showed similar results as compared to goniometer in terms of reliability, validity and accuracy of this method.

\section{Results}

The system of digital photography, non-reflective markers gave similar results for goniometer on the board. Three repeated tests for each angle in terms of mean are shown in Table 1.

Table 1. Values of three repeated tests for each given angle and the difference between each reference angle (absolute and relative error) and the average angles in terms of reference angle.

\begin{tabular}{|c|c|c|c|c|c|c|}
\hline Reference angles & Trial 1 & Trial 2 & Trial 3 & Average & Absolute error & Relative error \\
\hline 15 & 16.15 & 15.44 & 15.23 & 15.61 & -0.61 & 0.61 \\
\hline 30 & 31.53 & 31.55 & 31.38 & 31.49 & -1.49 & 1.49 \\
\hline 45 & 45.68 & 45.27 & 45.55 & 45.51 & -0.51 & 0.51 \\
\hline 60 & 59.74 & 59.94 & 60.14 & 59.95 & 0.05 & 0.05 \\
\hline 75 & 75.2 & 74.44 & 74.22 & 74.62 & 0.38 & 0.38 \\
\hline
\end{tabular}

Table 2. Validity $\left(R^{\wedge} 2\right)$, reliability (T-test, correlation) and accuracy (mean of absolute and relative error) of method of measuring angle.

\begin{tabular}{lllll}
\hline Repetitions & Validity $\left(\mathbf{R}^{\wedge} \mathbf{2}\right)$ & Reliability (T-test $\mathbf{p}$ value) & Reliability (correlation) & Absolute error \\
\hline $\mathrm{t} 1$ & 0.99 & 0.15 & 0.99 & - \\
\hline $\mathrm{t} 2$ & 0.99 & 0.24 & 0.98 & - \\
\hline $\mathrm{t} 3$ & 0.99 & 0.83 & 0.98 & - \\
\hline Mean & 0.99 & 0.41 & 0.97 & - \\
\hline
\end{tabular}

Validity of the system of digital photography, non-reflective markers was high $(<0.9)$ (Figure 2 ), and reliability of this system showed high correlation to traditional goniometer $(<0.9)$ and no significant difference between repetitions $(<0.05)$. Table 2 indicates values and mean of three tests. Absolute and relative error of above system is very low degree ( $>1$ degree) that indicates high accuracy of this system (Table 2).

\section{Discussion}

The objective of this study was to evaluate accuracy and the reliability of method comprising of non-reflective markers, Excel Software analysis and digital photography.

The reliability of the system with goniometer was 1 . This means that measurement in the limit of calibration board was consistent. 
Table 1 showed that the mean values of the measured angles were a little smaller than the references angles, Maximum differences were respectively $-1.49^{\circ}$ (for $30^{\circ}$ ), $-0.61^{\circ}$ (for $15^{\circ}$ ). $-0.51^{\circ}$ (for $45^{\circ}$ ), $0.05^{\circ}$ (for $60^{\circ}$ ) and $0.38^{\circ}$ (for $75^{\circ}$ ) for all angles, the error was from $-1.49^{\circ}$ to $0.38^{\circ}$. Error may be caused by the reflection of light but it was less than other studies (44, 43). This study had square markers which decreases the chance of error which may cause in previous studies as spherical markers had difficulty in finding corners [17].

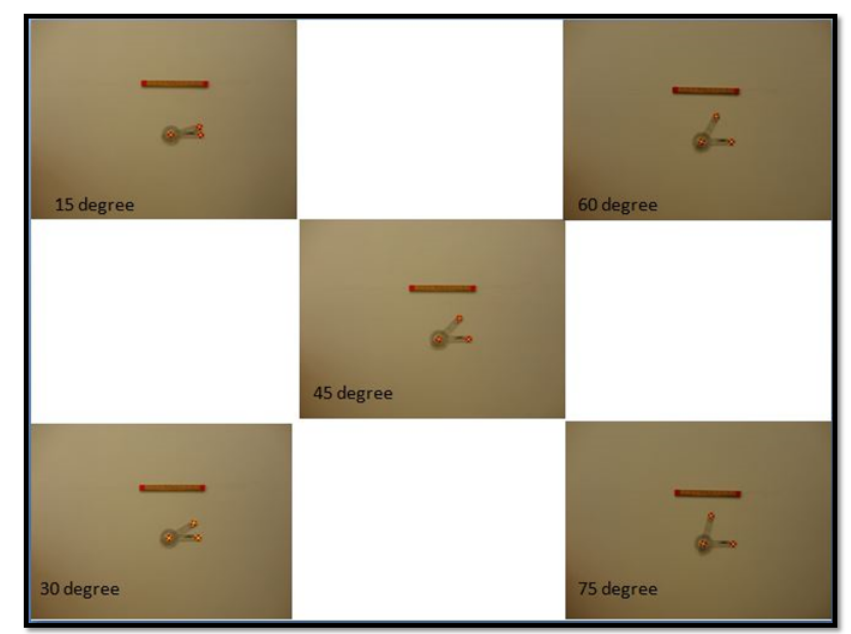

Figure 1. Goniometer with non-reflective markers shows at different angles $\left(15^{\circ}-75^{\circ}\right)$.

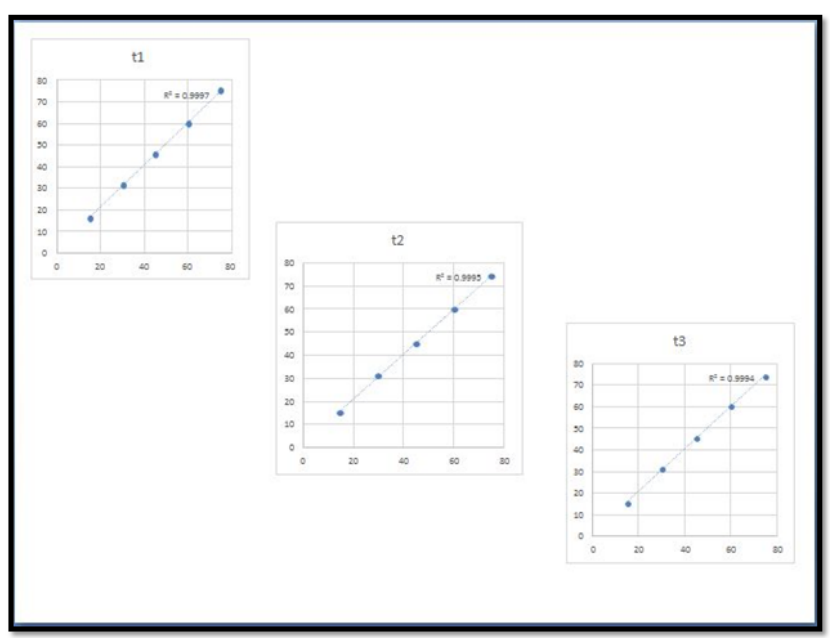

Figure 2. Showed the regression value for first, second and third trial which is 0.9997, 0.9995 and 0.9994.

This error does not exist in present method. The slopes of regression equations (validity test) were respectively 0.9997 , 0.9995 and $0.9994(\mathrm{P}=0.000)$ for all three repetitions. This means that this method was accurate to measure values of angles. Some previous studies used movie for measuring joint angle in which they recorded the angle through goniometer and protector, while measuring the angle they had to pause the video and then measure the angle on wall or wherever the projector reflects the light. This method causes many errors as surface where image was made may not smooth hinders in measurement. But this study used a new method which had no errors like previous studies [18]. Correlation coefficient in manual analysis of joint measurement was 0.9996 to 0.9999 $(\mathrm{P}=0.000)$. In this study researcher use contrast reflective color markers which also reduced the risk of error. Intra-rater reliabilities were high between the measurements respectively for all angles measured. In this study the goniometer was fixed on the wall to further decrease the risk of slipping and markers were used in square to make it easy to find the corners. It made the higher reliability of this method as compared to previous studies $[19,20]$. T-test also reinforced that it minimize the mean difference which is less as compared to previous studies [21]. Microsoft Excel and paint were the applications which rarely used in medical researches which were used in this study. Previous studies which used manual analysis showed that testretest reliabilities were high with manual calculation of errors but to calculate value from goniometer may cause some error as rounding off the value but Microsoft Excel is more precise in calculating the degrees as it set on reading 2 values after decimal [1,20,22-25].

Therefore this method is reliable to use in laboratory for measuring joint position sense. It is low cost and easy to use method. This study focus on points of previous studies which cause errors as using of square markers instead of circular, higher contrast markers, reflective markers, standard goniometer was used, camera was fixed on plain surface and light reflection was reduced by using high resolution camera and dark room $[17,26]$.

In conclusion, this was a novel approach to measure JPS with salient features of user friendly, fast, less expensive, accurate and reliable. Further researchers suggested using this novel method to measure JPS in weight bearing and non-weight bearing joints.

\section{Conflict of Interests}

We have no conflict of interests.

\section{Acknowledgment}

This is part of $\mathrm{PhD}$ thesis. This project was supported by Tehran University of Medical Sciences-International Campus, Faculty of Rehabilitation.

\section{References}

1. Karkouti E, Marks R. Reliability of photographic range of motion measurements in a healthy sample: knee and ankle joint measurement. Physiother Canada 1997; 49: 24-31.

2. Lattanzio PJ, Petrella RJ. Knee proprioception: a review of mechanisms, measurements, and implications of muscular fatigue. Orthopedics 1998; 21: 463-471.

3. Stillman BC, McMeeken JM, Macdonell RA. After effects of resisted muscle contractions on the accuracy of joint position sense in elite male athletes. Archiv Phys Med Rehab 1998; 79: 1250-1254.

4. Rome K, Cowieson F. A reliability study of the universal goniometer, fluid goniometer, and electrogoniometer for 
the measurement of ankle dorsiflexion. Foot Ankle Int 1996; 17: 28-32.

5. Clapper MP, Wolf SL. Comparison of the reliability of the orthoranger and the standard goniometer for assessing active lower extremity range of motion. Emory Univ 1986.

6. Stillman BC, McMeeken JM. The role of weight bearing in the clinical assessment of knee joint position sense. Aus J Physiother 2001; 47: 247-253.

7. Winter DA. Biomechanics and motor control of human movement. John Wiley \& Sons 2009.

8. Mueller MJ, Norton BJ. Reliability of kinematic measurements of rear-foot motion. Phys Ther 1992; 72: 731.

9. Clark NC, Akins JS, Heebner NR, Sell TC, Abt JP, Lovalekar M. Reliability and measurement precision of concentric-to-isometric and eccentric-to-isometric knee active joint position sense tests in uninjured physically active adults. Phys Ther Sport 2016; 18: 38-45.

10. Relph N, Herrington L. Interexaminer, intraexaminer, and test-retest reliability of clinical knee joint-position-sense measurements using an image-capture technique. J Phys Activity Health 2015.

11. Irving F, Russell J, Smith T. Reliability of knee joint position sense measurement: a comparison between goniometry and image capture methods. Eur J Physiother 2016; 18: 95-102.

12. Alhajaya MS. Effects of muscular fatigue on knee proprioception during performance and recovery of the knee flexors in female physical education students. Dirasat: Edu Sci 2016; 43.

13. Vander Linden DW, Carlson SJ, Hubbard RL. Reproducibility and accuracy of angle measurements obtained under static conditions with the motion analysis video system. Phys Ther 1992; 72: 300-305.

14. Scholz J. Reliability and validity of the WATSMART three-dimensional opto-electric motion analysis system. Phys Ther 1989; 69: 679-689.

15. Haggard P, Wing AM. Assessing and reporting the accuracy of position measurements made with optical tracking systems. J Motor Behavior 1990; 22: 315-321.

16. Sajjadi E, Olyaei GR, Talebian S, Hadian M-R, Jalaie S. The effect of forward head posture on cervical joint position sense. J Paramed Sci 2014; 5.
17. Vander Linden DW, Carlson SJ, Hubbard RL. Reproducibility and accuracy of angle measurements obtained under static conditions with the Motion AnalysisTM video system. Phys Ther 1992; 72: 300-305.

18. Stillman BC. An investigation of the clinical assessment of joint position sense. Univ Library 2000.

19. Jeng S-F, Schenkman M, Riley PO, Lin S-J. Reliability of a clinical kinematic assessment of the sit-to-stand movement. Phys Ther 1990; 70: 511-520.

20. Wilson DJ, Smith BK, Gibson JK, Choe BK, Gaba BC, Voelz JT. Accuracy of digitization using automated and manual methods. Phys Ther 1999; 79: 558-566.

21. Herrington L. Knee-joint position sense: the relationship between open and closed kinetic chain tests. J Sport Rehab 2005; 14: 356-362.

22. Foley CD, Quanbury AO, Steinke T. Kinematics of normal child locomotion-A statistical study based on TV data. J Biomech 1979; 12: 15-38.

23. Kadaba M, Ramakrishnan H, Wootten M, Gainey J, Gorton G, Cochran G. Repeatability of kinematic, kinetic, and electromyographic data in normal adult gait. J Orthop Res 1989; 7: 849-860.

24. Smith A. Variability in human locomotion: are repeat trials necessary? Aus J Physiother 1993; 39: 115-123.

25. Selfe J. Validity and reliability of measurements taken by the Peak 5 motion analysis system. J Med Eng Technol 1998; 22: 220-225.

26. Hadian M-R. Assessment of knee proprioception in the anterior cruciate ligament injury risk position in healthy subjects: A cross-sectional study. J Phys Ther Sci 2014; 26: 1515-1518.

\section{*Correspondence to}

Gholamreza Olyaei

School of Rehabilitation

Brain and Spinal Injury Research Center

Tehran University of Medical Sciences-International Campus (IC-TUMS)

Iran 